\title{
Detrás de las cifras de violencia contra las mujeres en Colombia ${ }^{1}$
}

\author{
Behind the Numbers of Violence Against \\ Women in Colombia
}

\section{Por trás das cifras de violência contra as mulheres na Colômbia}

\author{
María Alejandra García Otero ${ }^{2}$ \\ Centro Internacional de Agricultura Tropical, CIAT \\ Grupo Acción Colectiva y Cambio Social, ACASO \\ magarciaotero@gmail.com
}

\author{
María Eugenia Ibarra Melo³ \\ Profesora- investigadora Universidad del Valle \\ Grupo Acción Colectiva y Cambio Social, ACASO \\ maria.ibarra@correounivalle.edu.co \\ ORCID: 0000-0002-5667-6478
}

Recibido: 03/07/2016

Aprobado: $28 / 10 / 2016$

1 Este artículo presenta resultados del proyecto La respuesta institucional a la violencia contra las mujeres. Entre cifras y ficciones, desarrollado entre 2015 y 2016. Fue financiado por la Universidad del Valle y la Universidad de Los Andes en una convocatoria especial para apoyar la investigación con perspectiva de género, desde las Humanidades y el Derecho.

2 Socióloga, Magister en Sociología.

3 Socióloga, Doctora en Ciencias Sociales. 


\title{
Resumen
}

En este artículo se presenta un análisis de cómo algunas entidades gubernamentales construyen las cifras de violencia contra las mujeres en Colombia. Se describe el recorrido institucional de los datos hasta convertirse en las estadísticas que consultamos en los informes y boletines oficiales. Además, se analizan los conceptos en los que se fundamentan las mediciones y sus implicaciones en la comprensión del fenómeno. Más que las cifras, aquí interesan los instrumentos de captura y el modo en que aquellas se presentan. Por ello se recurrió al análisis documental y a la entrevista como principales herramientas de investigación. Se concluye que a pesar de los esfuerzos del Estado "de medir para controlar la violencia" y a quienes la perpetraron, es apremiante ajustar los procedimientos de captura de información para que sean más eficientes, sistemáticos y sensibles a la violencia que se comete contra las mujeres.

Palabras clave: violencia contra las mujeres; datos estadísticos; tipología de los datos.

\begin{abstract}
This article presents an analysis of how some government entities construct the statistical data of violence against women in Colombia. It describes the institutional course made by the data to become the statistics we query in reports and newsletters. In addition, it analyzes the concepts on which the measurements are based and their implications in understanding the phenomenon. More than the statistical data, here the interest is in the research instruments and the way in which they are presented. For this reason, documentary analysis and interviews were used as the main research tools. It is concluded that despite the efforts of the State to "measure to control violence" and those who perpetrated it, it is urgent to adjust the procedures for capturing information; and to be more efficient, systematic and sensitive to violence committed against women.
\end{abstract}

Key words: violence against women; statistical data; data typology.

\section{Resumo}

Este artigo apresenta uma análise da maneira como algumas entidades governamentais constroem os dados estatísticos da violência contra a mulher na Colômbia. Descreve-se o percurso institucional realizado com os dados até se tornarem em estatísticas publicadas em relatórios e boletins. Além disso, se analisam os conceitos que sustentam essas medições e suas implicações na compreensão do fenômeno. Mais do que os dados estatísticos, o mais importante são os instrumentos de pesquisa e a forma como eles são apresentados. Por essa razão, as principais ferramentas de pesquisa utilizadas foram a análise documental e as entrevistas. Conclui-se que, mesmo com os esforços do Estado em "medir para controlar a violência" e seus perpetradores, é urgente adequar os procedimentos de captura da informação para que sejam mais eficientes, sistemáticos e sensíveis à violência contra as mulheres.

Palavras-chave: violência contra as mulheres; dados estatísticos; tipologia de dados.

Este trabajo está bajo la licencia Creative Commons Attribution 3.0

¿Cómo citar este artículo? / How to quote this article?

García-Otero, M., y Ibarra-Melo, M. (2017, enero-junio). Detrás de las cifras de violencia contra las mujeres en Colombia. Sociedad y economía, (32), 41-64. 


\section{Introducción}

Hasta los años de 1980, la violencia contra las mujeres (VCM) se consideraba un problema personal. En la década de 1990 se convirtió en un problema social y en la actualidad se trata como un asunto público (Ibarra y García, 2012). Estos cambios en su definición se produjeron, en parte, por el activismo de las organizaciones feministas y de mujeres que reclamaban sus derechos al Estado, pero también por la presión que han ejercido los organismos internacionales para que estos sean garantizados y, por supuesto, porque hay respuesta institucional para responder a estas demandas (Almerás, Bravo, Milosavljevic, Montaño y Rico, 2002; Osborne, 2008; Roth, Guberek y Hoover, 2011; Almerás y Calderón, 2012).

De ese modo, la VCM se convirtió en un objeto de intervención estatal, que impone al Estado legislar sobre su prevención, atención y erradicación y disponer de un conjunto de instituciones que garanticen los derechos de las mujeres. No obstante, esa respuesta todavía no es satisfactoria. La atención ofrecida en los ámbitos nacional, departamental y local tiene amplias variaciones; las diferencias son notables entre la oferta institucional que reciben las ciudadanas en el ámbito urbano y las que habitan espacios rurales. También se presentan disparidades en el desarrollo de los sectores que contempla la Ley: salud, educación, trabajo y justicia.

Por lo anterior, la investigación realizada se interesaba en comprender ¿cómo se producen las estadísticas sobre la VCM en Colombia y qué agentes intervienen?, ¿qué implicaciones tienen las definiciones de la VCM en su medición?, ¿de qué modo las estadísticas que se producen sobre el asunto conducen a un tipo específico de políticas públicas, planes y programas para prevenir, atender y reducir la VCM? y ¿cómo interviene el Estado colombiano para prevenir, reducir y atender la VCM? Se trataba, entonces, de analizar la ruta de intervención que establece el Estado para atender un asunto que compromete la garantía de los derechos de las mujeres. Esta ruta empieza con la promulgación de normas, sigue con la generación de conocimiento sobre el asunto a través del sistema de indicadores de monitoreo y seguimiento y la respuesta judicial a determinados casos, y termina con la recolección de estadísticas de atención a las víctimas que acuden a los servicios de salud y a las entidades que administran justicia.

En este artículo solo daremos cuenta de la reconstrucción del proceso de producción de las estadísticas sobre la VCM en Colombia e identificamos a los agentes que intervienen en él. En ese sentido nos propusimos: a) describir el recorrido institucional que permite la captura, sistematización y análisis de datos e b) Identificar los condicionamientos sociales que influyen en el proceso. Lo anterior con el fin de contribuir a mejorar los procesos de medición de la VCM. Tratamos de mostrar las implicaciones que tienen las definiciones de la VCM en su medición y el modo en que las estadísticas estatales contribuyen a visibilizar el fenómeno en los medios de comunicación, la academia, el movimiento social y en los organismos internacionales, agencias, consultores y ONG. 
El proceso es de doble vía: de un lado, las estadísticas permiten interpretar y relacionar la dinámica de la VCM y, de otro lado, las denuncias del movimiento feminista y de mujeres, a través de distintas formas de acción colectiva, presionan a las instituciones para que midan el fenómeno, incorporando nuevos elementos que detectan en la transformación del fenómeno. Estas demandas se acompañan con la presión que ejerce la comunidad internacional sobre el Estado colombiano, para que cumpla con pactos y acuerdos firmados; de los resultados de la investigación académica; de las intervenciones del tercer sector para atender a las víctimas; así como de la difusión de las noticias en los medios. Como consecuencia de esto, las mediciones contribuyen a la formulación de políticas públicas, planes, programas, y campañas para prevenir, atender y reducir la VCM.

El punto de inicio de esta investigación se estableció en la promulgación de la Ley 1257 de 2008, por la cual se establecen normas de sensibilización, prevención y sanción de formas de discriminación contra las mujeres ${ }^{4} \mathrm{y}$ se analizaron registros hasta el 2014.

\section{La estrategia de investigación}

Este proyecto combinó distintas técnicas y procedimientos de investigación sociológica y jurídica. La más importante fue el análisis de contenido de leyes, decretos, políticas públicas, formularios y bases de datos de las distintas entidades que se enuncian en la siguiente sección, así como la revisión de prensa. Como complemento de esta información, se realizaron entrevistas a funcionarios del Instituto Nacional de Medicina Legal y Ciencias Forenses (INMLCF), Profamilia (representantes de la Encuesta Nacional de Demografía y Salud -ENDS-) y de la Fiscalía General de la Nación. Se revisaron 76 documentos producidos por instituciones, la mayoría de carácter público (5 fueron de carácter privado), correspondientes a los informes del INMLCF (51 documentos) y los boletines del Observatorio de Asuntos de Género de la Presidencia de la República. El corpus documental se construyó con 38 boletines estadísticos, 31 informes de resultados, 5 instrumentos de registro y 2 conferencias. A estos documentos se les aplicó un formulario ${ }^{5}$ de preguntas relacionadas con su caracterización general, se extrajeron de ellos los conceptos utilizados de VCM, tipo de víctimas, victimarios y escenarios de violencia medidos, tipo de análisis realizado, entre otros aspectos relacionados con los objetivos de investigación.

El formulario correspondió a la operacionalización de las siguientes dimensiones: caracterización de los documentos, taxonomía (tipos de violencias medidas, víctimas, victimario y escenarios), uso de la cuantificación (objetivo del estudio, propósito, tipo de análisis, herramientas técnicas usadas, etc.) y dimensión sociológica (profesionales participantes, referencia a normatividad nacional e internacional y de otros sectores de la sociedad). Este se aplicó a los documentos, como si se tratara de una "encuesta", para ello se utilizó como herramienta el programa Atlas.ti, especial para el tratamiento de información cualitativa.

4 Entre otras disposiciones, la Ley determina las instancias encargadas de atender y generar sistemas de registro e información respecto a la VCM.

5 El instrumento es un formulario utilizado en el trabajo de investigación de María Alejandra García (2015). 


\section{La estructura del artículo}

El artículo se estructura en cinco secciones. La primera ofrece una descripción de las instituciones que miden la violencia contra las mujeres. La segunda presenta el recorrido institucional de las cifras de VCM y un análisis del proceso de construcción de las estadísticas, registros administrativos y la elaboración de estudios empíricos -encuestas-. La tercera parte muestra el modo en que se recopila información sobre VCM mediante registros administrativos; para ello utilizamos como ejemplos representativos los casos de la Fiscalía y el INMLCF. En la cuarta, presentamos el modo en que se recolecta la información a través de estudios cualitativos y cuantitativos para conocer mejor el comportamiento de la VCM, un proceso que hemos llamado análisis de subsuelo. La quinta sección hace énfasis en los condicionamientos sociales del proceso de construcción de las cifras de VCM.

\section{Instituciones gubernamentales que miden la violencia contra las mujeres}

El Estado colombiano mide la VCM, principalmente, mediante dos procedimientos a) la administración de datos individuales del registro de denuncias y muertes y b) la realización de estudios empíricos. Esta responsabilidad recae en entidades que, por su actividad cotidiana, tienen el compromiso y la posibilidad de hacerlo; aunque su misión principal no sea registrar datos sobre hechos que se configuran como VCM. Como sugiere Desrosiéres (2007), estas formas de registro administrativo son características de las fuentes de información que generan los Estados.

En este caso, la agregación de los datos recae en las siguientes dependencias ${ }^{6}$ : el Instituto Nacional de Medicina Legal y Ciencias Forenses (INMLCF), que tiene como finalidad mostrar la evolución de los homicidios, los suicidios y las lesiones personales de toda la población colombiana; la Unidad Nacional de Víctimas, que recopila las denuncias de las víctimas y les otorga un registro que los acredita como beneficiarios para acceder a la reparación; y la Encuesta Nacional de Demografía y Salud (ENDS), que tiene como objetivo mejorar e institucionalizar la recolección y utilización de la información requerida para evaluar los programas de salud sexual y salud reproductiva y de población (Profamilia, 2011)7.

6 De acuerdo con el Sistema Nacional de Vigilancia en Salud Pública (Sivigila), del Instituto Nacional de Salud (INS), entre las violencias basadas en género se encuentran la física, psicológica, la negligencia y abandono, el abuso sexual, el acoso sexual, la violación, la explotación sexual comercial de niños niñas y adolescentes, la trata de personas para explotación sexual, la violencia sexual en el conflicto armado y los actos sexuales violentos. El INMLCF produce estadísticas sobre violencia intrafamiliar, delito sexual, lesiones personales y homicidios. Desde 2009, introduce los conceptos de femicidio, feminicidio y homicidio en mujeres. Sobre el primero señala que se refiere a una "muerte violenta de una mujer, perpetrada por un hombre por factores asociados al género y que puede darse en espacios privados o públicos" (Acero, 2009, p. 25). El Registro Único de Víctimas, de la Unidad Nacional de Víctimas, no tiene un hecho victimizante que permita dar cuenta de la violencia contra las mujeres. No obstante, entre los hechos que registra aparecen los Delitos contra la libertad y la integridad sexual, diferenciados por sexo.

7 Este instrumento concentra su foco de atención en mujeres en edad reproductiva (15 a 49 años) y excluye a un buen número de mujeres que también sufren la violencia sexual en Colombia, como las niñas entre o y 14 años y las mayores de 50. 
Los datos del INMLCF ofrecen una amplia cobertura de casos y valiosa información sobre homicidios, suicidios y lesiones personales. Para los casos de VCM, el Instituto incluye en sus informes distintas variables que permiten comprender mejor las circunstancias de los hechos violentos que se presentan en determinados años. Por ejemplo, para el período que abarca esta investigación (2008-2014), se brindan estadísticas acerca de la violencia sociopolítica, intrafamiliar, común impulsiva y económica, y desde 2010 se incorpora el delito sexual. Respecto a los homicidios, las cifras se desagregan según grupo vulnerable, sexo y nivel de escolaridad de la víctima, presunto agresor, escenario del hecho. Para el posible delito sexual, los datos se presentan según escenario del hecho y agresor, desagregados por sexo. El capítulo de violencia intrafamiliar muestra las tasas de maltrato de pareja según edad y sexo, escolaridad y ocupación de la víctima.

Otras entidades que resguardan grandes volúmenes de información son la Policía Nacional y el Instituto Nacional de Salud (INS). La primera solicita a la Unidad Operativa Interpol-DIJIN y al Centro de Investigaciones Criminológicas (Cicri) el procesamiento, la consolidación y difusión de los registros administrativos, con fines estadísticos de delitos y actividad operacional, públicos desde el 2010. Anualmente reporta datos sobre homicidios, delitos sexuales, lesiones personales y violencia intrafamiliar, entre otros. El proceso de registro se inicia con la ejecución de un hecho o acción considerada como delito que llega a conocimiento de la Policía por distintas fuentes: formales (denuncias o querellas instauradas en las salas de denuncias, salas de atención al usuario o centros de servicios judiciales) o informales (escritos y llamadas anónimas, medios de comunicación y de otro tipo). Los reportes estadísticos tienen como finalidad asesorar al mando institucional en el direccionamiento del servicio de Policía y contribuir en la formulación de la política pública. En los registros se discrimina las víctimas por sexo, aparece el escenario del hecho y el arma o medio utilizado, pero no se registra quién fue el agresor ni su sexo.

El INS registra cifras de violencia a través del Sivigila por intermedio de la Subdirección de Vigilancia y Control en Salud, responsable de definir e implementar el modelo operativo del Sistema de Vigilancia y Control en Salud Pública en el Sistema General de Seguridad Social en Salud. Entre sus funciones se contempla el desarrollo de parámetros para la vigilancia de diferentes eventos de interés en salud pública. Para el caso que interesa en esta investigación, la entidad lo denomina violencias de género. Esta institución justifica la vigilancia de los eventos de violencia, al considerarlos un problema de salud pública, dado que ellos producen daños en la salud física y emocional de las víctimas, sus familias y la sociedad. Otra razón para el seguimiento de este fenómeno es la cantidad de población afectada (niñas, niños, adolescentes, jóvenes y mujeres de todas las edades). "El propósito de la vigilancia es realizar monitoreo continuo y sistemático con el fin de contribuir al diseño de las políticas públicas para la prevención y la intervención oportuna de sus consecuencias" (Ortiz y Forero, 2015, p. 12).

La notificación del evento la realiza un profesional de salud, de la institución prestadora de salud (IPS) pública o privada, determinando si el caso cumple con la definición de violencia de género establecida en el protocolo y la aplicación de la ficha de notificación. De esa parte se encargan las unidades primarias 
generadoras de datos. Después, la información llega a las secretarías municipales de salud y luego a las secretarías distritales y departamentales y al INS. Si bien esta información es pública, las fichas de notificación no están disponibles en su página $w e b$, argumentando la confidencialidad del dato8.

Por su parte, el registro único víctimas (RUV) reporta los casos de mujeres agredidas por un actor armado, legal o ilegal, en el marco del conflicto armado, pero excluye los casos de violencia que se producen en situaciones diferentes a la confrontación. Esta base de datos tiene mayor precisión en la fecha de registro del hecho ante la institución, que en fecha de ocurrencia del delito. A pesar de la magnitud de la violencia que han desplegado los actores armados contra las mujeres, en Colombia estas estadísticas son muy dispersas y tienen amplia variabilidad. Los datos sobre VCM se ocultan en los grandes agregados de victimización por desplazamiento forzado, detenciones arbitrarias, tomas de rehenes y reclutamiento forzado de sus hijos y familiares, los atentados a sus organizaciones y a sus líderes, los crímenes de ejecución extrajudicial, las desapariciones forzadas, la tortura física, psicológica y sexual, las pérdidas materiales, entre otras.

También existen otros sistemas que proporcionan datos de interés, pero que no dependen directamente del Estado. Desafortunadamente, estos datos no están disponibles en línea.

(...) Por ejemplo, el Sistema de Gestión de Datos sobre Violencia Basada en Género (GBVIMS), una iniciativa interagencial de la Oficina del Alto Comisionado de Naciones Unidas para los Refugiados (ACNUR), el Fondo de Población de las Naciones Unidas (UNFPA), el Fondo de Naciones Unidas para la Infancia (UNI$\mathrm{CEF}$ ), la Organización Mundial de la Salud (OMS), y el Comité Internacional del Rescate (IRC). El GBVIMS facilita a los prestadores de servicios médicos, judiciales y psicosociales en contextos humanitarios recolectar, guardar y analizar datos de incidentes de violencias basadas en género, VBG, reportados, utilizando herramientas y definiciones estandarizadas para recolectar y compartir información sobre estos incidentes de manera ética, segura y anónima. El objetivo del GBVIMS es diseñar mecanismos de prevención de VBG con base en las estadísticas que él mismo genera, además de garantizar a las víctimas/sobrevivientes de estas violencias una atención integral y de calidad. El GBVIMS se aplica en catorce países del mundo. En Colombia, se implementa en siete municipios piloto, desde octubre de 2011, en Barrancabermeja, Santander; Medellín y Apartadó en Antioquia y desde junio de 2012 en Arauca, Arauca; Buenaventura, Valle del Cauca; Tumaco, Nariño, y Villavicencio, Meta (UNFPA y ACNUR, 2012, p. 7).

Como se pudo comprobar en esta investigación, en Colombia son varias las entidades gubernamentales que albergan cifras de violencia contra las mujeres, mediante la identificación de asuntos sociales de interés con datos desagregados por sexo. Algunos se concentran en violencia intrafamiliar, violencia sexual, y en otros se enfatiza en comprender la violencia contra las mujeres o la violencia

8 "La ficha de notificación es para fines de vigilancia en salud pública y todas las entidades que participen en el proceso deben garantizar la confidencialidad de la información según lo dispuesto en la Ley 1273 de 2009 para la protección de la información y bases de datos y la ley estatutaria 1266 de 2008 por la cual se dictan las disposiciones generales del hábeas data y se regula el manejo de la información contenida en bases de datos personales" (Ortiz y Forero, 2015, p. 12). 
de género. A continuación, se describe en detalle cómo las entidades en mención construyen las estadísticas de violencia contra las mujeres en Colombia.

\section{Recorrido institucional de las cifras de VCM}

Más allá de juzgar si estas estadísticas son insuficientes para abordar las dimensiones que ha tomado la VCM en Colombia, en el presente artículo destacamos los aportes que las instituciones antes nombradas proveen para profundizar en su comprensión. Como se deduce de los objetivos de este estudio, aquí no interesan tanto las cifras, sino cómo se producen y de qué modo son utilizadas por los usuarios de las estadísticas (instituciones, incluidos los juzgados y las Cortes, investigadores y académicos, consultores, ONG, organismos internacionales y agencias de cooperación, que realizan intervenciones con víctimas, los medios de comunicación y otros agentes). Adicionalmente a las cifras que estas instituciones producen, son muy importantes los informes que realizan, basados en el método epidemiológico, propio de la salud pública. Uno de los más importantes es el Forensis datos para la vida, que se presenta como una herramienta para la interpretación, intervención y prevención de lesiones de causa externa en Colombia. En este se presentan estadísticas, reflexiones y recomendaciones realizadas por expertos de distintas áreas del conocimiento (economía, sociología, psicología, derecho, etc.)

En este sentido, coincidimos con Pringle y Watson (2002) y damos importancia al estudio de las prácticas que construyen al Estado, en vez de tomar como dadas sus estructuras. Para estas autoras, el Estado no refleja simplemente las desigualdades de género, sino que, a través de sus prácticas, desempeña una importante función en la constitución de esas desigualdades. Simultáneamente, las prácticas de género se institucionalizan en formas estatales históricamente específicas. Es una vía de doble sentido.

Otras contribuciones de la sociología y la historia de la estadística (Hacking, 1975; Desrosiéres, 1996; 2004; 2007) y la sociología de la cuantificación (Espeland and Stevens, 2008) recuerdan la importancia de entender las estadísticas públicas como construcciones socio-históricas que hacen parte de la conformación del Estado, así como las prácticas de funcionarios y los lineamientos de la ciencia.

Igualmente, las contribuciones clásicas de Weber (1977), o de autores contemporáneos como Bourdieu (2005) y Gupta (2012), señalan que las prácticas burocráticas se relacionan con los procesos de construcción de estadísticas o cifras de asuntos públicos. Las discusiones teóricas que introducen estos autores delimitan la importancia de los aspectos sociales, históricos, de construcción del Estado y las intermediaciones subjetivas en las cifras administrativas. Estos aspectos también han sido tratados en estudios más circunscritos al análisis de la construcción de indicadores de género (Scott, 1999) o indicadores de VCM, ya sea para dilucidar los aspectos que se debe mejorar (Ferrer, Bosch y Riera, 2006; Casado, García y García, 2012); para institucionalizar la medición de VCM (Roth et al., 201l; Almerás et al., 2002; Fries y Hurtado, 2010; Almerás y Calderón, 2012) o para generar conocimiento al respecto (Castro y Riquer, 2003; Daza y Pérez, 2008; Osborne, 2008).

Para el presente estudio se dio prioridad a las entidades estatales que más producen cifras sobre VCM. Estas son el INMLCF, el Ministerio de Salud y Protección 
Social (mediante la ENDS), la Fiscalía General de la Nación, el Observatorio de Asuntos de Género de la Presidencia de la República (OAG), la Defensoría del Pueblo y el Observatorio de Derechos Humanos de la Presidencia (ODDHH) 9

Estas entidades realizan procesos administrativos y burocráticos para informar, hacer monitoreo y seguimiento a la frecuencia, incremento y evolución del número de víctimas de los tipos de violencia clasificada en la Ley 1257 de 2008. En estos procesos se entrecruzan aspectos característicos de un Estado racional y moderno, en el cual la centralización, unificación, estandarización, eficiencia (uso del tiempo e incorporación de tecnologías de información) y prácticas humanas juegan un rol importante para dar cuenta de la realidad. Sin embargo, en esa supuesta imparcialidad y objetividad de los datos producidos por el Estado, también interfieren valoraciones subjetivas de los funcionarios que participan en la construcción de las estadísticas públicas sobre VCM. Este último aspecto se trata someramente en esta investigación ${ }^{10}$.

A continuación, se describen brevemente dos formas en que las entidades construyen un hecho social y lo presentan en cifras de VCM: registros administrativos y recolección de información primaria (ej. encuestas y estudios de caso). Se pone especial énfasis en los cuatro pasos que componen el proceso: captura, registro, reporte y publicación de la información. Se tienen en cuenta aspectos centrales tales como la capacidad instalada del Estado para llevar a cabo el procesamiento de datos estadísticos (infraestructura física, tecnológica y de recurso humano) y la taxonomía sobre VCM y conceptos relacionados. Y claro está que entran en estos aspectos el contexto normativo nacional e internacional que denominamos condicionamientos sociales, los cuales no se expondrán por ahora. La taxonomía hace referencia a la forma en que las entidades estatales, por medio de los documentos públicos, clasifican y codifican los hechos sociales. En otras palabras, a la manera en que se definen los conceptos claves para la descripción del hecho social, que en este caso es VCM. Esto último se hizo mediante el análisis de contenido al que fue sometido el corpus documental seleccionado, con la construcción de un formato que captura y categoriza las formas en que el Estado entiende la VCM.

\section{Registros administrativos}

En la captura de la información primaria, mediante registros administrativos, trabajan principalmente el INMLCF y la Fiscalía General de la Nación. Esta se inicia con la visibilidad de los hechos de violencia (física y sexual) y los homicidios que son cometidos contra las mujeres, que luego son denunciados por ellas o sus familiares ante las instancias correspondientes. Este proceso empieza cuando una víctima acude a un centro de salud, una estación de la Policía o una sede de la Fiscalía, para denunciar un acto de violencia, según las rutas de

9 De ningún modo olvidamos que otras entidades también miden este asunto público.

10 Quizás en un estudio posterior se incorporen análisis sociológicos más profundos, que contribuyan a disminuir el sesgo valorativo de esas intromisiones en la construcción de los datos. Un primer intento de medir la percepción de los funcionarios acerca de este fenómeno es la Medición sobre tolerancia institucional de las violencias contra las mujeres, de la cual se han realizado dos ejercicios, el último en 2015. 
atención creadas en la normatividad nacional, o a partir de un caso de asesinato que llega al conocimiento de la Fiscalía y/o al INMLCF. Este procedimiento previo condiciona la calidad de la información.

Por ello, como afirma el INMLCF (2008), el subregistro es una debilidad que no se ha podido superar en las estadísticas sobre VCM en Colombia. Para esta entidad, los datos no logran dilucidar las agresiones verbales, físicas, sexuales y psicológicas que ocurren en los hogares, lugares de trabajo, centros educativos y espacios públicos y que no son denunciadas ni por las víctimas, ni por los testigos. Entre otras razones, el subregistro se sigue presentando porque "se denuncian menos hechos de los que acontecen realmente; o porque puede haber desistimiento previo a la valoración médico-forense" (INMLCF, 2009b, p. 9). Por lo anterior, en los informes de estas entidades se habla de casos registrados.

En este punto se ubica una de las principales dimensiones del análisis que proponemos en esta investigación: la relación entre el Estado y la víctima, que tiene origen en el recorrido que emprende una mujer cuando acude a las rutas de atención institucional establecidas para las víctimas de violencia ${ }^{11}$. El primer registro legaliza la relación, se identifica e individualiza el caso y se incluye en las cifras que reportan las dependencias en sus informes y boletines. No obstante, como se ha señalado antes, esa relación solo es posible si las mujeres denuncian o si las autoridades registran los homicidios declarados. Es decir que el reconocimiento estatal de la VCM todavía pasa por la voluntad de las víctimas para notificar los hechos ante las instituciones competentes o ante la obligación institucional de reportar la ocurrencia de un hecho fatal (la muerte). De ahí se desprenden esas "fuentes primarias de información" que otras entidades estatales (como la Defensoría del Pueblo, OAG y el ODDHH) usan para promover la protección de los derechos de las mujeres, difundir la problemática y generar discusiones acerca de cómo restituir derechos, mejorar los servicios de atención, entre otros derivados del uso de las cifras por parte del Estado.

Esta primera identificación puede verse afectada por varios aspectos, en especial en los casos de homicidio. Tal como expone el INMLCF, hay varias circunstancias que contribuyen al subregistro de casos e inciden en la identificación de hechos de violencia física y sexual. Por ejemplo, cuando en el proceso de inspección previo al análisis del cadáver no se registra la suficiente información sobre el contexto del hecho; cuando se realizan manipulaciones indebidas del cuerpo (asunto tratado a partir de una estandarización de la cadena de custodia); cuando se acelera el proceso natural de descomposición del cuerpo o la experticia o especialización del médico son insuficientes para realizar los dictámenes. Según un reporte del INMLCF (2009b), "los médicos suelen estar ciegos y solos con el cuerpo en sus salas" (p. 340). Estas situaciones se presentan "especialmente en los casos en que médicos rurales y oficiales de hospitales cubren el servicio de peritaje donde el INMLCF no tiene presencia directa, lo que se refleja en los

11 Para más información sobre la ruta de atención en salud se recomienda leer el trabajo de grado: López, M. (2016). "Los golpes maltratan el cuerpo, pero a ellas también les duele el alma" Reflexiones sociológicas sobre la atención en salud a la violencia contra las mujeres. (Trabajo de grado en Sociología). Universidad del Valle, Cali, Colombia. 
reportes que escriben" (García, 2015, p. 54) ${ }^{12}$. Por lo anterior, el INMLCF ha implementado estándares internacionales, tales como el Protocolo de Minnesota de 1991, para que los médicos forenses mejoren la identificación de los casos de violencia sexual en cadáveres.

Retomando el análisis de la relación víctima-Estado, es indispensable señalar que la captura de información hace parte de esa relación. Es por ello que las cifras de VCM están marcadas por las rutas de atención establecidas por el Estado para atender a las víctimas. En otras palabras, el proceso de operacionalización de los tipos de violencia, consignados en conceptos jurídicos de la Ley 1257 de 2008 a indicadores cuantitativos de VCM, ha sido posible porque esta ley también define rutas y protocolos de atención para las entidades. Se trata de un paso, como se señaló atrás, que depende de la voluntad de la víctima para denunciar el hecho. Con este acto se inicia el proceso de medición tal como se muestra en la tabla l, elaborada a partir de la información documental y de las entrevistas realizadas para esta investigación.

Tabla 1. Proceso administrativo de construcción de estadísticas públicas de violencias contra las mujeres. Colombia. 2007-2013

\begin{tabular}{|c|c|}
\hline Entidad estatal & Pasos administrativos \\
\hline INMLCF & $\begin{array}{l}\text { 1. Una víctima inicia un proceso de denuncia o se inicia el reporte de un } \\
\text { asesinato. } \\
\text { 2. Un asistente de clínicas recibe una solicitud de un examen médico } \\
\text { legal, de una entidad competente (que haga parte de la ruta de } \\
\text { atención). } \\
\text { 3. Un médico autorizado hace una valoración médico forense y escribe un } \\
\text { reporte. } \\
\text { 4. Un asistente o el médico registra información del caso, datos } \\
\text { generales de la víctima y el dictamen final del examen en sistemas de } \\
\text { información centralizados (por ejemplo, el aplicativo en línea Siclico } \\
\text { del INMLCF para la sistematización de la información y análisis de } \\
\text { violencia en Colombia). } \\
\text { 5. La información reposa en un sistema centralizado en Bogotá. } \\
\text { 6. Se consolida la información en Excel y es depurada. } \\
\text { 7. Se hace un reporte estadístico mensual, como datos preliminares. } \\
\text { 8. Se hace un informe anual, Forensis, con datos del Instituto y opiniones } \\
\text { de analistas. }\end{array}$ \\
\hline $\begin{array}{l}\text { Fiscalía General } \\
\text { de la Nación }\end{array}$ & $\begin{array}{l}\text { 1. Denuncia de la víctima por medio de la ruta de atención. } \\
\text { 2. Se hace un registro de la denuncia en un sistema web. } \\
\text { 3. Quien recibe la denuncia hace una solicitud a INMLCF para un } \\
\text { examen médico forense y remite al grupo de psicólogos de la Fiscalía. } \\
\text { 4. Atención en el grupo de psicólogos, reporte de la sesión y registro de } \\
\text { información en la web. } \\
\text { 5. Registro de datos generales de los atendidos. } \\
\text { 6. Centralización de la información en Bogotá. }\end{array}$ \\
\hline
\end{tabular}

Fuente: Elaboración propia a partir de entrevistas semiestructuradas a funcionarios y revisión de documentos estatales de 2007 a 2014.

12 A propósito de este inconveniente, el propio INMLCF señaló las deficiencias de 968 municipios del país que no tienen morgue y donde las necropsias se realizan sin las condiciones sanitarias adecuadas (Medina, 2016, p. 4). 
Un segundo paso es el registro de la información. A partir de los casos de VCM, ambas entidades inician un proceso de registro, en el que se clasifican los tipos de violencias que usualmente son entendidas como VCM, de acuerdo con sus definiciones. Por ejemplo, la violencia intrafamiliar (incluye la de pareja), la violencia sexual y el homicidio son desagregadas por el sexo de la víctima y partir de ello construyen las estadísticas públicas sobre el asunto.

Ese proceso, dependiendo de la entidad, puede ser paralelo a la captura de información o continuo durante varios procesos administrativos. Las entidades analizadas cuentan con tecnologías de información que permiten: a) tener un formulario estándar para caracterizar el hecho con variables específicas (identificación de la víctima -sexo, etnia, nombre, orientación sexual-, lugar de ocurrencia, presunto victimario, móvil, lesiones de la víctima), y b) centralizar la información en una base en línea a la que tienen acceso las sedes centrales en Bogotá. Sin duda, esto posibilita la sistematización del proceso, la centralización de la información y la eficiencia en el reporte de datos para la toma de decisiones. Un ejemplo de lo anterior lo proporciona una funcionaria del INMLCF: "Si quisiéramos, podemos hacer la consulta a hoy de cuántos casos hemos recibido de la atención clínica por cualquiera de los contextos por violencia" (Coordinadora del Centro de Referencia, comunicación personal, 15 de junio de 2015).

El sistema web del INMLCF, que almacena la información de atención a personas vivas (incluye los casos de violencia intrafamiliar, sexual, física, entre otras), recibe el nombre de Siclico. Es una herramienta para el análisis de la violencia en el país y es definido por esta entidad como un "Sistema de Información de Clínica Forense, en el que se está realizando la captura de variables epidemiológicas actualizadas de acuerdo con requerimientos de leyes como la 1257 y la ley de víctimas, entre otras, aplicando los reglamentos técnicos médico legales vigentes" (INMLCF, 2016). Permite capturar y almacenar información nacional en tiempo real, para que investigadores y tomadores de decisiones puedan analizarla.

La información de las personas desaparecidas y fallecidas se almacena en el Sistema de Información Red de Desaparecidos y Cadáveres (SIRDEC). Estas plataformas virtuales permiten centralizar en tiempo real todos los hechos identificados a escala nacional, en la sede central en Bogotá; también se ha creado un tercer sistema, el Sistema de Vigilancia Epidemiológica de Lesiones de Causa Externa (Sivelce), que recopila toda la información en un solo lugar. En esta parte de sistematización y organización de la información participan ingenieros de sistemas que hacen control de calidad de la información, sin modificar los datos suministrados por profesionales especializados de la salud encargados de la captura de los datos. Esto hace parte de los procesos de racionalización del Estado, donde la estandarización y centralización son cruciales.

En un tercer momento se reporta la información, a partir de los parámetros de la estadística descriptiva para mostrar cuadros de frecuencias absolutas y relativas. Estas entidades no analizan la información, solo reportan datos. No obstante, las publicaciones que realizan son escritas por psicólogos, sociólogos (especialistas en políticas sociales), estadísticos (especialistas en análisis de datos), comunicadores, abogados, politólogos, economistas, médicos y especialistas en epidemiología, criminalística y manejo de sistemas de información. "Se trata de 
incorporar los aportes de estas disciplinas y sus especialidades para un mejor procesamiento de la información, profundidad de los análisis y presentación de los datos" (García, 2015, p. 56).

Usualmente, se registra y maneja la información del caso, teniendo en cuenta variables demográficas de la víctima (sexo, edad, escolaridad, ocupación, estado civil, etnia o pertenencia a grupos vulnerables -discapacidad física, desplazados-) y contexto del hecho (la actividad que estaba realizando la víctima cuando ocurrió el hecho -labores domésticas, estudiaba, trabajaba-), móvil de la violencia, fecha, hora y lugar. Las clasificaciones de los hechos, además de tener en cuenta estas variables, sufren modificaciones a través del tiempo, que cambian las estadísticas de algunos tipos de VCM. Por ejemplo, en 2008, el INMLCF incluyó los casos de violencias cometidos por la expareja de la víctima en la categoría de violencia de pareja y la violencia a personas mayores fue creada como una subcategoría de la violencia intrafamiliar. Otro aspecto que también influye en la construcción de estadísticas en el INMLCF es que se carece de un sistema de clasificación o información que registre hechos como violencia patrimonial y psicológica.

Por último, esta información se utiliza para la toma de decisiones gubernamentales internas. Es decir, en la Fiscalía se utilizan estos datos para iniciar procesos judiciales y el INMLCF comparte esta información con sus dependencias internas y con otras entidades estatales. Este último publica los datos en la serie anual Forensis, que trata la violencia intrafamiliar y la sexual en acápites especiales. Adicional a ello, en una sección especial publica artículos relacionados, pero escritos por personas externas al Instituto. Algunos de estos polemizan con las cifras del Instituto y generan debate sobre la información publicada, también la contrastan con otras fuentes de información no oficiales, cuantitativas o cualitativas, recolectadas de manera independiente por organizaciones civiles.

\section{Recolección de información}

Las entidades estatales son conscientes del subregistro en las actuales estadísticas de VCM. Para contrarrestar esta dificultad realizan estudios de carácter cualitativo y cuantitativo que les permitan recolectar los datos que quedan ocultos ante el sistema de información estatal. A este proceso lo denominamos análisis de subsuelo, porque pretende hurgar en los resquicios donde pocas veces llega la administración estatal (García, 2015). De este proceso hacen parte los estudios público-privados, como la Encuesta Nacional de Demografía y Salud (ENDS), liderada por Profamilia, pero financiada y apoyada técnicamente por el Ministerio de Salud y Protección Social, y por organismos internacionales. También están los estudios del Observatorio de Asuntos de Género, que indagan por casos particulares, por ejemplo: violencia contra mujeres afrodescendientes.

Este proceso de recolección de información tiene similitudes con el anterior, en la medida en que hay pasos de recolección, procesamiento, análisis y publicación de la información. Pero, así como se muestra en la tabla 2, hay divergencias claras en el paso a paso de estos procesos, especialmente en la construcción y procesamiento de los casos identificados de VCM. 
Tabla 2. Proceso de construcción de estadísticas públicas de violencias contra las mujeres mediante recolección de información. Colombia. 2007-2013

\begin{tabular}{|c|c|}
\hline $\begin{array}{c}\text { Entidad estatal o } \\
\text { estudio }\end{array}$ & Pasos administrativos \\
\hline ENDS & $\begin{array}{l}\text { 1. Negociaciones presupuestales } \\
\text { 2. Definición de metodología (diseño muestral) } \\
\text { 3. Revisión y diseño de instrumentos de investigación } \\
\text { 4. Entrenamiento en la metodología (coordinadores de campo } \\
\text { 5. Recolección de información } \\
\text { 6. Depuración de información } \\
\text { 7. Reporte de datos } \\
\text { 8. Informe final }\end{array}$ \\
\hline $\begin{array}{l}\text { OAG (estudios } \\
\text { cualitativos) }\end{array}$ & $\begin{array}{l}\text { 1. Formulación de objetivo } \\
\text { 2. Revisión de fuentes de información disponibles } \\
\text { 3. Construcción de una metodología } \\
\text { 4. Levantamiento de información } \\
\text { 5. Análisis } \\
\text { 6. Publicación de resultados }\end{array}$ \\
\hline
\end{tabular}

Fuente: Elaboración a partir de entrevistas semiestructuradas a funcionarios y documentos estatales de 2007 a 2014.

Aunque la ENDS se enfoca en aspectos demográficos, de salud, y salud sexual y reproductiva, uno de sus módulos profundiza en la violencia intrafamiliar. En el 2015 abarcó la violencia de género y consultó tanto a hombres como a mujeres. La ejecución de esta encuesta, que data de 1995 y se aplica cada cinco años, conlleva cinco pasos. El primer paso es la definición y consecución de recursos para realizarla; hecho importante, porque de ello depende el alcance y la representatividad estadística del estudio. Paralelamente, se diseña la muestra, para la cual es preciso definir, junto con los financiadores, el alcance del estudio y la unidad de análisis/sujetos de análisis. Durante muchos años la encuesta fue representativa solo a escala nacional y únicamente eran entrevistadas mujeres en edad reproductiva. Para la última, que se ejecutó en el 2015 (aún no hay resultados), la muestra permite la representatividad departamental y nacional. En esta se entrevistaron los hombres del hogar, lo que permite mejorar los análisis y formular recomendaciones de política más efectivas en lo local.

En el segundo paso, se construyen los cuestionarios de la investigación. Estos constan de instrumentos de información que recolectan datos del hogar y otros que van dirigidos explícitamente a mujeres en edad reproductiva. En el 2010 se hicieron "cinco cuestionarios [...] l. Cuestionario de hogar para ser aplicado en cada hogar visitado. 2. Cuestionario individual para mujeres en edad fértil (1349 años). 3. Cuestionario sobre prevención de cáncer de cuello uterino y mama (mujeres 18-69 años). 4. Cuestionario de adulto mayor, para hombres y mujeres de 60 años y más. 5. Cuestionario de peso y talla, para niños menores de cinco años y sus madres" (Profamilia, 2011, p. 7).

Para su diseño, el grupo de investigación de Profamilia hace un análisis de las lecciones aprendidas. Revisa las preguntas formuladas a lo largo del tiempo y los cambios realizados a otras. Este análisis es realizado según los temas en los que 
se divide la encuesta. En el 2015 se consultó por: antecedentes de la entrevistada; reproducción, anticoncepción, embarazo, parto y posparto, educación sexual, nupcialidad y exposición al riesgo de embarazo, preferencias de fecundidad, trabajo de la mujer, detección temprana de cáncer, VIH/sida y otras infecciones de transmisión sexual (ITS); violencia de género (llamada violencia intrafamiliar en las anteriores encuestas) y roles de género (capítulo nuevo).

Tercero, cada uno de los módulos es entregado a expertos en el tema, que organizan y proporcionan sugerencias de preguntas nuevas en pro de mejorar el módulo. No obstante, los expertos no están autorizados a introducir preguntas y variables que no hayan sido aplicadas en otras encuestas nacionales o internacionales. Como menciona la directora de investigaciones de Profamilia: "nosotras no diseñamos preguntas, aprobamos o no el ingreso o la salida de alguna pregunta" (funcionaria de Profamilia, comunicación personal, 16 de junio de 2015).

La construcción del cuestionario continúa con la consolidación de una mesa de expertos constituida por integrantes de Profamilia, ONG, organismos internacionales, organizaciones civiles y el Gobierno nacional. En esta mesa se valida la calidad de las preguntas de cada uno de los módulos, desde un trabajo intersectorial, en el que se destaca la presencia del Departamento Administrativo Nacional de Estadística (DANE), ONU Mujer, Ministerio de Salud y Protección Social, el Fondo de Población de la ONU y académicos de diversas instituciones.

Listo el cuestionario final en su versión impresa, se pasa a un software utilizando la tecnología CAPI (computer-assisted personal interviewing), que permite recolectar la información en línea. Posteriormente, se hace una prueba piloto en uno de los municipios no seleccionados en la muestra, con el objetivo de probar las preguntas, categorías de respuesta y el flujo de cada uno de los módulos.

El cuarto paso es la capacitación de encuestadores (tabla 2). Este inicia con la contratación del personal, que en su mayoría tiene experticia en la aplicación de formularios de salud. Antes de 2015, todas las encuestadoras y coordinadoras de campo eran mujeres, puesto que no se entrevistaba hombres. Esto cambió para la última encuesta.

El quinto paso es la captura de la información, que sigue rigurosamente el cuestionario prediseñado. Durante este paso se llevan a cabo varios controles de calidad para evitar los errores humanos. Las verificaciones se hacen desde el mismo día de la aplicación de la encuesta. Una de las ventajas del software de información en línea, es que la encuesta es enviada en tiempo real a la base central, ubicada en Profamilia-Bogotá. Allí se inicia la revisión de la coherencia de la información, controlando minuciosamente la calidad de los datos registrados para evitar que se afecte la representatividad de la muestra. Acto seguido se depura la información cuando toda la base de datos está completa (tabla 2). En este proceso participan, especialmente, ingenieros de sistemas y otros expertos.

Con la base limpia, se inicia la tabulación de los resultados, que se basan en estadísticas descriptivas ya preestablecidas por Profamilia, y permiten seguir el tipo de análisis realizado en versiones anteriores. El reporte de estadísticas de esta etapa impide que los profesionales cambien el enfoque del análisis; de nuevo hay un control en el uso de la información en pro de la calidad de la investigación y del cumplimiento de estándares internacionales. Esta información se entrega a los expertos en cada tema para que escriban un capítulo sobre el 
módulo correspondiente, de acuerdo con los lineamientos de interpretación de datos proporcionado por Profamilia. El informe se publica en la página web de la ENDS y es compartido con tomadores de decisiones y organizaciones civiles, que hacen uso de la información para la formulación de políticas adecuadas a la realidad. No obstante, una funcionaria de Profamilia hace hincapié en que no toda la información recolectada se hace pública por su gran volumen, pero que la institución responde a las solicitudes de datos que realizan entidades civiles, académicas y gubernamentales (funcionaria de Profamilia, comunicación personal, 16 de junio de 2015).

En el siguiente diagrama (1) se puede observar un resumen de los pasos que llevan las cuatro entidades a partir de las cuales se ha hecho principalmente el análisis en los párrafos anteriores.

Diagrama 1. Resumen de los pasos en registros administrativos y recolección de información primaria

Registros administrativos

\begin{tabular}{|c|c|c|c|c|c|c|}
\hline $\begin{array}{c}\text { Denuncia de } \\
\text { la victima }\end{array}$ & $\begin{array}{c}\text { Funcionarios } \\
\text { reciben la solicitud } \\
\text { y ejecutan } \\
\text { procedimientos }\end{array}$ & $\begin{array}{l}\text { Registro de la } \\
\text { información en } \\
\text { sistemas de } \\
\text { información }\end{array}$ & $\begin{array}{c}\text { Procedimientos } \\
\text { de atención y } \\
\text { completar } \\
\text { información }\end{array}$ & $\begin{array}{c}\text { Información } \\
\text { cenralizada en } \\
\text { Bogotá }\end{array}$ & $\begin{array}{l}\text { Análisis de } \\
\text { información }\end{array}$ & $\begin{array}{l}\text { Reporte de } \\
\text { información }\end{array}$ \\
\hline
\end{tabular}

Recolección de información primaria

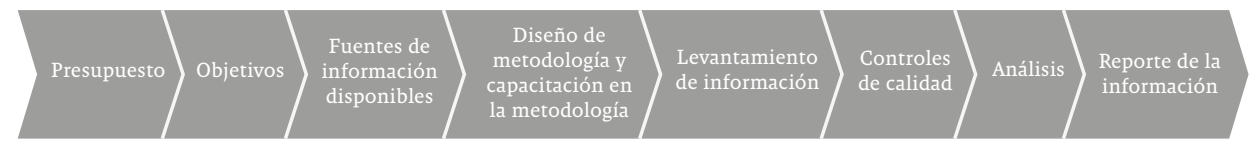

Fuente: Elaboración a partir de entrevistas semiestructuradas a funcionarios y documentos estatales de 2007 a 2013.

Se observa en general que las estadísticas provenientes de registros administrativos dependen en parte de las denuncias sobre hechos de violencia contra las mujeres y de las diferentes atenciones que la entidad brinda a la víctima. En el caso del INMLCF están los procedimientos médico-legales y en la Fiscalía el proceso legal. Por su parte, las cifras que hacen parte de la recolección de información dependen del presupuesto que se designe desde el Estado para la elaboración de estudios, las metodologías a usar y los procedimientos de recolección de información. Como se observa en el siguiente punto, ambos procedimientos están influenciados por condicionamientos sociales.

\section{Condicionamientos sociales}

El proceso administrativo de recolección de información o la ejecución de estudios empíricos para obtener cifras de VCM, ya sea con enfoque cuantitativo o cualitativo, está mediado por condicionamientos sociales. Estos incluyen desde el contexto normativo nacional e internacional, pasando por la capacidad de los recursos tecnológicos y de conocimiento experto que posean las instituciones, hasta por la taxonomía que el Estado plasma en sus documentos sobre VCM y 
el ser mujer. En lo que concierne a este estudio, profundizamos en los siguientes condicionamientos sociales: recurso humano, capacidad instalada y recursos tecnológicos, perspectiva sobre el uso de las cifras y taxonomía.

\subsection{Recurso humano}

Las entidades analizadas cuentan con recurso humano que procede de distintas ramas del conocimiento. Estos actores se diferencian por los campos del saber que dominan y por el rol administrativo que cumplen en la burocracia estatal. Los profesionales más destacados en estos procesos son los médicos y epidemiólogos, los psicólogos, politólogos, sociólogos, economistas, ingenieros de sistemas y los técnicos administrativos.

Los profesionales de la salud se dedican a dictaminar la ocurrencia de la violencia sexual o física en los cuerpos vivos o fallecidos de las presuntas víctimas. Estos se encuentran en el INMLCF y los centros de salud. Durante el periodo de análisis, se pudo comprobar que estos profesionales recibieron formación por parte de instancias estatales o del tercer sector para enfrentar su trabajo. No obstante, la revisión documental permite establecer que sus prácticas para identificar algunos casos de VCM depende también de su experiencia en el tema, del número y la calidad de las capacitaciones que hayan recibido (INMLCF, 2009a). Por ejemplo, en el INMLCF los funcionarios recibieron entrenamiento técnico de los protocolos de Minnesota y Estambul, y en la Fiscalía en la atención de hechos de violencia sexual a menores de edad.

Estos profesionales tienen un rol protagónico en el levantamiento de la información. Ellos utilizan variables de los análisis epidemiológicos: ocurrencia del hecho, características de la víctima y del victimario, momento del suceso y móvil. El papel crucial que cumplen estos profesionales da indicios de cómo entiende el Estado la violencia contra las mujeres, es decir, como lo configura en delito y de qué modo lo asume cuando lo considera un problema de salud pública.

Por su parte, los ingenieros se dedican al procesamiento y centralización de la información, haciendo uso de sistemas tecnológicos para capturar, procesar y analizar la información nacional en tiempo real. Por ello, su papel es importante en Profamilia, el INMLCF y la Fiscalía. Los estadísticos se encargan del "procesamiento de los datos, control de calidad a nivel "científico", determinando errores de muestreo y significancia" (García, 2015, p. 76).

Los especialistas en ciencias sociales, economistas, sociólogos y politólogos, se centran en el análisis de los datos y escritura de los documentos que publican estas entidades; por ejemplo de Forensis, el principal medio de difusión del INMLCF. Estos profesionales también participan en el diseño metodológico de las investigaciones de la ENDS y el OAG, sobre todo en el proceso de recolección de información, ya sea como encuestadores, entrevistadores y/o coordinadores de campo.

Durante el proceso de recolección, registro y análisis de información es importante resaltar el papel de los psicólogos. Por ejemplo, en la Fiscalía el grupo de psicólogos de las seccionales centrales atiende a quienes denuncian hechos de violencia sexual o intrafamiliar y complementa la información de las víctimas con el dictamen impartido por INMLCF. Este informe se convierte automáticamente en una prueba jurídica de la que puede disponer el juez para tomar 
decisiones sobre el caso. En el INMLCF, los psicólogos participan en el análisis de la información y redacción de documentos.

Si bien en este estudio no se profundiza en las valoraciones de los funcionarios y en la incidencia de estas en el proceso de medición, reconocemos que es un asunto de gran importancia. Al respecto Desrosiéres (2004), desde la sociología de la estadística, y Gupta (2012), desde la antropología del Estado, han señalado la necesidad de entender las prácticas de los funcionarios, porque estas componen y representan la práctica burocrática que involucra la construcción de cifras de violencias. Varios análisis sociológicos han puesto en relieve cómo la práctica burocrática puede reflejar un proceso de racionalización del Estado o, por el contrario, intersecciones de valoraciones subjetivas (Weber, 1977; Bourdieu, 2005).

El propio INMLCF muestra que la calidad de datos y la identificación de hechos de violencia están condicionados por la escasa conciencia o el poco conocimiento de la situación, que tienen algunos médicos o quienes hacen el registro de información. Los funcionarios de la Fiscalía entrevistados reconocieron que algunos profesionales se rehúsan a utilizar los sistemas tecnológicos para captura de información, porque siguen prefiriendo el papel, lo que genera retrasos en el registro de datos y mayores errores en el procesamiento de datos. Otro ejemplo de cómo incide la subjetividad en la medición o en la atención de la VCM, se puede observar en los resultados del estudio de la Presidencia de la República de Colombia que consultó a 1.095 funcionarios públicos y encontró altos grados de tolerancia con la VCM. Como señalan sus autores, ello incide en la debida atención de los casos y en la revictimización de quienes denuncian (Proyectamos Colombia, 2015).

A la par, otros ejemplos muestran que los funcionarios aplican procedimientos estándares y se alinean con los marcos normativos que son estipulados por las entidades nacionales o internacionales. Un ejemplo de ello son los reglamentos existentes en la Fiscalía para seguir las clasificaciones del Código penal, los cuales no admiten interpretaciones más amplias de las solicitadas. En el mismo sentido, aparecen los lineamientos fijados por Profamilia para el análisis de la ENDS, o del INMLCF para la publicación en Forensis. Estos dejan escaso margen de maniobra a los analistas para incorporar criterios diferentes a los establecidos.

Con base en lo anterior, se puede afirmar que la formación académica y la subjetividad de los funcionarios contribuye a producir el proceso de medición de la VCM y, de ese modo, afectan la construcción de las cifras. Las valoraciones de estos profesionales en el momento de registro de información y atención a la víctima, como el modo en que ejecutan los lineamientos de práctica burocrática estándar modifican los datos. Adicionalmente, el uso de nuevas tecnologías para el manejo de información, ha sido muy importante en este proceso.

\subsection{Capacidad instalada y recursos tecnológicos}

En los últimos años, las instituciones estatales han racionalizado sus prácticas, ya sea en el proceso administrativo de recolectar datos o en el levantamiento de información primaria (ej. ENDS). En dicho proceso es fundamental la implementación de tecnologías de información que permiten mayor centralización 
de los datos recolectados a lo largo del país; una homogeneización de los instrumentos de recolección de información y un control de calidad.

El uso de este software ha comprimido el tiempo y el espacio. Ahora, mientras el médico forense de cualquier seccional del país introduce datos en un instrumento único nacional sobre el examen pericial de una víctima de VCM, los funcionarios administrativos de Bogotá pueden consultar este y los demás casos en tiempo real. Sin duda, esto es un indicador del proceso de racionalización del Estado.

La pluma, la tinta, el papel y los archivos documentales han cedido paso a los registros digitales almacenados en internet. Ellos dejan menor cabida a la interpretación y opinión escrita de funcionarios, especialmente en la primera etapa de la recolección de información. Estas tecnologías garantizan, en parte, menor intervención humana en un proceso que se supone objetivo y se supone que disminuyen los errores en la captura del dato.

Cada vez más, estas instituciones incorporan en sus procesos operativos sistemas de información, software (SAS, STATA, SPSS) y tecnologías, como la CAPI, entre otros requerimientos básicos para la recolección sistemática y análisis de grandes volúmenes de datos que se obtienen con las encuestas, como la ENDS o los registros de casos que captura el INMLCF. Estas son herramientas necesarias para que el Estado construya cifras sobre VCM en Colombia.

\subsection{Perspectiva sobre los datos y taxonomía}

Las propuestas analíticas desde la sociología de la estadística (Desrosiéres, 2004) señalan la importancia de entender la taxonomía relacionada al asunto de medición. Es decir, de qué manera se entiende y se clasifica el concepto de VCM y de otros conceptos relacionados (sujetos de medición y lugares en que se perpetúa la violencia), en el contenido de los documentos estatales que publican las cifras de este asunto público.

En las entidades estatales que se tienen en cuenta en este estudio, la taxonomía en las cifras de VCM se divide en dos grandes grupos. El primero, la taxonomía de enunciación, que se refiere a aquellas clasificaciones de VCM que son nombradas y conceptualizadas en los documentos, pero que carecen de una concreción estadística, es decir, no son medidas. Un ejemplo, son aquellas categorías abstractas de violencia de género y VCM. A este nivel, Osborne (2008) lo denomina nivel conceptual. Estas son definidas a partir de lo dictado por la normatividad nacional. Es por ello que la definición más recurrente de VCM se toma de la Ley 1257 de 2008:

\footnotetext{
Cualquier acción u omisión, que le cause muerte, daño o sufrimiento físico, sexual, psicológico, económico o patrimonial por su condición de ser mujer, así como las amenazas de tales actos, la coacción, o la privación arbitraria de la libertad, bien sea que se presente en el ámbito público o privado.
}

Aún falta en el quehacer administrativo una operacionalización que logre traducir estas definiciones en indicadores medibles regularmente.

En la taxonomía de enunciación también se incorporan aquellos tipos de VCM reconocidos por el Estado, pero que no son objeto de medición. Verbigracia, 
está "la prostitución forzada, desplazamiento forzado, reclutamiento forzado, violencia económica y feminicidio" (García, 2015, p. 84). Estos conceptos no son referentes normativos en la legislación nacional y solo se los trata como tipos de violencia porque los autores de los análisis hacen referencia a ellos, no porque el Estado produzca cifras constantes de los mismos.

El segundo gran grupo lo integran los conceptos de VCM que constituyen la taxonomía de medición, la cual es conceptualizada y medida de manera reiterativa o esporádica. De manera sistemática, aquí aparece la violencia sexual, intrafamiliar y de pareja. De forma esporádica, se puede encontrar la violencia verbal, física, psicológica, patrimonial, el homicidio y el suicidio. El primer subgrupo lo constituyen aquellas violencias que el Estado mide y construye cifras a partir de su quehacer administrativo. Las segundas, versan entre la taxonomía de la enunciación y la medición, pues son medidas en estudios específicos o en apartados especiales de los informes más representativos, como Forensis.

Las tres taxonomías más importantes se caracterizan por cuatro características centrales. La primera, porque hay varios términos a partir de los cuales se entiende dicha violencia como subtipos que la componen; es decir, no hay consenso sobre el término apropiado para denominar el hecho y a su vez estos subtipos de VCM se dividen en otros. Por ejemplo, para violencia sexual las entidades estatales utilizan diferentes referentes teóricos para distintas maneras en que se da este subtipo de violencia. Es el caso del abuso sexual (acto sexual de un mayor a un menor), asalto sexual (el victimario persigue un propósito sexual definido hacia una persona que no tiene el consentimiento ni la voluntad de hacerlo), acceso carnal violento (penetración del miembro masculino o de cualquier objeto en cualquier parte del cuerpo sin el consentimiento de la persona), violencia sexual homicida (homicidios que suceden cuando se está llevando a cabo un hecho de violencia sexual), prostitución forzada y violación sexual. Para la violencia intrafamiliar y de pareja pareciera haber más consenso, pero no es así. Para la primera, la variación está entre violencia intrafamiliar y violencia entre familiares y para la segunda entre violencia de pareja, violencia física de pareja y violencia contra la pareja.

La segunda característica es que son violencias que desmitifican la idea de la dicotomía de lo público-privado. La violencia intrafamiliar, de pareja y sexual, son aquellas que ocurren en un contexto de interrelaciones personales o lo que tradicionalmente se ha considerado "privado". Pero las instituciones, al considerar estas violencias un asunto público y generar cifras al respecto, afirman que estas no son de la esfera privada, sino de la esfera pública y, por ello, deben ser medidas. No obstante, esta desmitificación no es completamente satisfactoria, si tenemos en cuenta los principios de equidad de género y los aportes de algunas propuestas feministas. Estas violencias son las que tradicionalmente han sido identificadas con la VCM y asumen que todas las víctimas son madres, esposas, parejas y objetos sexuales. Dejan por fuera múltiples tipos de violencias que se dan en otros contextos, donde las mujeres cumplen otros roles (ej. violencia verbal en la esfera laboral).

Implícitamente, se proporcionan cifras para aquellas violencias en contextos "privados", bajo el supuesto que ese es el lugar que ocupan las mujeres. Entonces, hay una clase de doble proceso: se desmitifican las violencias privadas para 
mostrarlas en cifras públicas, pero no se profundiza en otros tipos de violencias que ocurren en espacios públicos. Este fenómeno se relaciona, en parte, con el proceso de construcción de normatividad jurídica nacional, pues cabe recordar que por un buen tiempo en Colombia la VCM se entendió como violencia intrafamiliar (incluida la de pareja) y violencia sexual (Ibarra y García, 2012). En las cifras actuales, aún quedan vestigios de ese antecedente histórico.

Un tercer elemento, es que estas tres violencias se entienden, por lo menos desde los informes de resultados analizados, como producto de relaciones de poder en que las mujeres se encuentran en una posición de desventaja en el marco de un contexto patriarcal. Esto es de suma importancia, puesto que por lo menos en el contenido escrito, hay un reconocimiento a los aportes del movimiento feminista y a los desarrollos de las teorías de género. Ya la VCM no es un hecho producto de una "enfermedad mental o psicológica" de una persona que acomete un acto violento contra una mujer; sino que es parte de las relaciones desiguales de género. Un avance sumamente importante en términos de entender la VCM como un asunto público y un objeto de cuantificación.

Una última característica fundamental de los principales conceptos de la taxonomía de medición es que la categorización se basa en quien perpetúa el hecho. Conocer al victimario y la relación con la víctima es fundamental para determinar el tipo de violencia (intrafamiliar, de pareja, sexual, etc.). Por ello, es que se distingue la violencia sexual contra la pareja, una de las violencias "más ocultas" y la violencia intrafamiliar -perpetrada por una persona que tiene una relación cercana con la víctima-. En este punto es importante tener en cuenta que las relaciones afectivas entre el victimario y víctima son de importancia para las cifras de la VCM. Por lo anterior, el INMLCF introdujo la violencia ejercida por la expareja como parte de la violencia intrafamiliar y constituyente de la categoría violencia contra la pareja. No obstante, a pesar de la prevalencia de la caracterización de estos victimarios, también se ha empezado a categorizar aquellos que no tienen una relación con la víctima, por ejemplo, quienes pertenecen a un grupo armado legal o ilegal. Sin duda un avance importante para determinar el impacto de la violencia contra las mujeres en el marco del conflicto armado.

Estas violencias en cifras, la intrafamiliar, sexual y de pareja, en la actualidad parecieran ser más datos de violencia desagregados según el sexo de la víctima que mediciones propiamente de VCM, teniendo en cuenta la conceptualización que se da en la Ley 1257 de 2008 , es decir, un acto que se comete contra las mujeres por el hecho de ser mujeres. Hasta el momento, no se diferencian las violencias que se producen en razón del género de la víctima de aquellas en que el acto de violencia se produce exclusivamente por otros móviles. Este punto es sumamente importante, pues muestra que aún falta una mejor operacionalización de algunos conceptos legales, la taxonomía de la enunciación y las cifras de VCM. Atenderlo contribuiría a visibilizar aquellas VCM que no se reducen al ámbito privado o a una relación afectiva entre la víctima y el victimario.

\section{Reflexiones finales}

En Colombia existen entidades estatales que mediante su quehacer administrativo recopilan datos desagregados de violencia según el sexo de la víctima, 
que son entendidos como casos de VCM o de algunos de sus tipos: violencia intrafamiliar, sexual o de pareja. Estas cifras se construyen en el marco de un proceso racional caracterizado por su centralización, parametrización de instrumentos de registros y recolección de información, división del trabajo de acuerdo con las áreas de conocimiento de los funcionarios y uso de tecnologías para el registro y análisis de la información en un menor tiempo y con mayor control de calidad. Pero a la vez, es un proceso que tiene intermediaciones subjetivas, precisamente por las prácticas de los funcionarios y sus valoraciones en torno a la VCM.

En ese proceso interfieren condicionamientos sociales que se relacionan con el recurso humano que trabaja en las instituciones estatales, la tecnología que han incorporado y, sobre todo, las definiciones con las que se clasifica la VCM. Como se observó en este proceso de investigación, la VCM está dividida entre una taxonomía de enunciación y medición, un proceso que ha desmitificado la "armonía" de ciertos espacios y relaciones afectivas, pero que aún no logra diferenciar entre cifras de violencia en que las víctimas son mujeres y las cifras de VCM. Por lo anterior, es apremiante una mejor operacionalización de lo que Osborne (2008) denomina conceptos de VCM y las cifras de violencia.

Más que juzgar las cifras de VCM, en este artículo se destacan las contribuciones institucionales y las dimensiones influyentes en el proceso de construcción de datos. No obstante, se resaltan algunos aspectos que deberían tenerse en cuenta para mejorar la acción estatal. En primer lugar, ampliar las tipologías de VCM medidas, desarrollando procesos de generación de información en cuanto a la violencia patrimonial y psicológica, estipuladas por la Ley y reconocidas por la comunidad académica. Mejorar la coordinación dentro de las instituciones y entre ellas para sistematizar con mayor precisión los casos de VCM y así contribuir a la reducción del subregistro. Ajustar los procedimientos de captura de información, para que sean aún más eficientes, sistemáticos y sensibles al género.

Por lo anterior, consideramos que el Estado colombiano tiene el reto de incorporar mediciones más amplias de la VCM, incluyendo la violencia que acontece en el mundo rural y en el marco del conflicto armado, puesto que los principales desarrollos hasta ahora se han concentrado en la violencia urbana. En parte, por la infraestructura que requieren estos procesos de captura, procesamiento y análisis de la información. Así mismo, es indispensable que la academia utilice más y mejor estos datos y contribuya a comprender la información que producen estas instituciones, a la luz de la diversidad de las mujeres, incluyendo a aquellas que viven en el "resto" (área rural), a las mujeres mayores, a las negras e indígenas y a las trans.

Por último, consideramos que es importante iniciar investigaciones que incorporen la diferencia entre cifras de violencias desagregadas según el sexo de las víctimas, y violencias contra las mujeres por razones de género.

\section{Referencias}

Acero, A. P. (2009). Homicidio, 2009. Aproximaciones a los conceptos de femicidio, feminicidio y homicidios en mujeres. Bases para su medición. En INMLCF (Eds.), Forensis (pp. 18-68). Bogotá D.C., Colombia: División de referencia de información pericial - CRNV. 
Almerás, D., Bravo, R., Milosavljevic, V., Montaño, S. y Rico, M. N. (2002). Violencia contra la mujer en relación de pareja: América Latina y El Caribe. Una propuesta para medir su magnitud y evolución. CEPAL-Serie Mujer y desarrollo, (40), 4-54.

Almerás, D. y Calderón, C. (2012). Cuando los datos hablan. Información sobre la violencia contra las mujeres. En D. Almerás y C. Calderón (Coords.), Si no se cuenta, no cuenta. Información sobre la violencia contra las mujeres (pp. 31-119). Santiago, Chile: Organización de las Naciones Unidas, CEPAL.

Bourdieu, P. (2005). De la casa del rey a la razón de Estado: un modelo de la génesis del campo burocrático. En L. Wacquant (Coord.), El Misterio del Ministerio (pp. 71-80). Barcelona, España: Gedisa.

Casado, E., García, A. A. y García, F. (2012). Análisis crítico de los indicadores de violencia de género en parejas heterosexuales en España. Empiria. Revista de metodología de Ciencias Sociales, (24), 163-186.

Castro, R. y Riquer, F. (2003). La investigación sobre violencia contra las mujeres en América Latina: entre el empirismo ciego y la teoría sin datos. Saude Pública, 19(1) 135-146.

Daza, S. y Pérez, T. (2008). Contando mujeres. Una reflexión sobre los indicadores de género y ciencia en Colombia. Antropol. sociol., (10), 29-51.

Desrosiéres, A. (1996, enero 17 al 19). Reflejar o instituir: La invención de los indicadores estadísticos. Presentado en Los indicadores sociopolíticos hoy. Jornadas de estudio. Observatorio Interregional de lo Político y Asociación Francesa de Ciencia Política. París, Francia.

Desrosiéres, A. (2004). La política de los grandes números. Barcelona, España: Melusina.

Desrosiéres, A. (2007). Surveys Versus Administrative Records: Reflections on the Duality of Statistical Sources. Courrier des statistiques, (13) 7-19.

Espeland, W. N. \& Stevens, M. L. (2008). A Sociology of Quantification. European Journal of Sociology, 49(3), 401-436.

Ferrer, V., Bosch, E. y Riera, T. (2006). Las dificultades en la cuantificación de la violencia contra las mujeres en la pareja: análisis psicosocial. Intervención psicosocial, 15(2), 181-201.

Fries, L. y Hurtado, V. (2010). Estudio de la información sobre la violencia contra las mujeres en América Latina y El Caribe. Serie: CEPAL- Mujer y desarrollo, (99), 2-57.

García, M. A. (2015). Tras los bastidores de las estadísticas de la violencia contra las mujeres en Colombia. Un análisis desde la Sociología de la cuantificación (tesis de maestría). Universidad del Valle, Cali, Colombia.

Gupta, A. (2012). Red Tape: Bureaucracy, Structural Violence and Poverty in India. Durham, US: Duke University Press.

Hacking, I. (1975). El surgimiento de la probabilidad. Un estudio filosófico de las ideas tempranas acerca de la probabilidad, la inducción y la inferencia. Barcelona, España: Gedisa.

Ibarra, M. E. y García, M. A. (2012). La violencia contra las mujeres: un asunto público. La Manzana de la Discordia, 7(2), 23-34.

Instituto Nacional de Medicina Legal y Ciencias Forenses (INMLCF). (2008). Forensis. Datos para la vida. Herramientas para la interpretación, intervención y 
prevención de lesiones de causa externa en Colombia. Bogotá D.C., Colombia: División de referencia de información pericial - CRNV.

Instituto Nacional de Medicina Legal y Ciencias Forenses (INMLCF). (2009a). Forensis. Datos para la vida. Herramientas para la interpretación, intervención y prevención de lesiones de causa externa en Colombia. Bogotá D.C., Colombia: División de referencia de información pericial - CRNV.

Instituto Nacional de Medicina Legal y Ciencias Forenses (INMLCF). (2009b). Masatugó "Mujer que recibe lo malo, para entregar lo bueno" 2004-2008. Forensis Mujeres. Datos para la vida. Herramienta para la interpretación, intervención y prevención de lesiones de causa externa en Colombia para las mujeres. Bogotá D.C, Colombia: División de referencia de información pericial - CRNV.

Instituto Nacional de Medicina Legal y Ciencias Forenses (INMLCF). (2016). SICLICO: una herramienta para el análisis de la violencia en Colombia. Recuperado de http://www.medicinalegal.gov.co/-/ siclico-una-herramienta-para-el-analisis-de-la-violencia-en-colombia

Ley 1257 de 2008. (4 de diciembre de 2008). Diario oficial No. 47.193, 2008, 4, diciembre.

Medina, L. (16 de febrero de 2016). Casi 9 de cada 10 municipios no tienen morgue. El Tiempo, p. 4.

Ortiz, M. y Forero, L. J. (2015). Protocolo de vigilancia de salud pública. Violencia de género. Bogotá, Colombia: Instituto Nacional de Salud.

Osborne, R. (2008). De la violencia (de género) a las "cifras de la violencia": una cuestión política. Empiria. Revista de metodología de las Ciencias Sociales, (15), 99-124.

Pringle, R. y Watson, S. (2002). "Los intereses de las mujeres" y el Estado postestructuralista. En M. Barrett y A. Phillips (Comps.), Desestabilizar la teoría. Debates feministas contemporáneos (pp. 67-89). México D.F., México: Universidad Nacional Autónoma de México, Paidós.

Proyectamos Colombia. (2015). Segunda medición del estudio sobre tolerancia institucional de las violencias contra las mujeres. Informe final. Bogotá, Colombia: Proyectamos Colombia.

Profamilia. (2011). Encuesta Nacional de Demografía y Salud. ENDS 2010. Bogotá, Colombia: Profamilia.

Roth, F., Guberek, T. y Hoover, A. (2011). El uso de datos cuantitativos para entender la violencia sexual relacionada con el conflicto armado colombiano: retos y oportunidades. Bogotá D.C., Colombia: Corporación Punto de vista.

Scott, J. (1999). El mundo del trabajo a través de las estadísticas. La estadística de la industria en París (1847-1848). En J. Scott. (Ed.), Género e Historia (pp. 148-177). México D.F., México: Fondo de Cultura Económica.

UNFPA y ACNUR. (2012). Implementación del sistema de gestión de datos sobre violencias basadas en el género-GBVIMS en Colombia. Bogotá, Colombia: UNFPA-ACNUR.

Weber, M. (1977). Economía y sociedad. México D.F., México: Fondo de Cultura Económica. 